\title{
TENDENCIAS EN EL USO DE ESPECIES EN PLANTACIONES DE PEQUEÑOS Y MEDIANOS PROPIETARIOS PERÍODO 2012-2016
}

\author{
Avila, Alberto y Muñoz, Juan Carlos ${ }^{10}$
}

\section{RESUMEN}

La superficie de plantaciones forestales en Chile alcanza en 2015 a 2,4 millones de hectáreas y la tasa anual de plantación se mantiene por sobre las 90 mil hectáreas, tasa que hoy está representada fundamentalmente por la reforestación (reposición de plantaciones cosechadas), dado que desde el año 2012, cuando expiró la ley de fomento a la forestación (plantaciones en suelos sin cubierta forestal), esta cayó drásticamente. En el año 2016 la reforestación alcanzó a 96.043 ha en tanto que la forestación a solo 2.421 ha.

Las principales especies en las plantaciones del país a 2015 son Pinus radiata, Eucalyptus globulus y Eucalyptus nitens, con 1.400 .259 ha, 576.937 ha y 259.299 ha, respectivamente. La diferencia respecto del total está dada por 147.434 ha de plantaciones de otras especies, de géneros como Pinus, Pseudotsuga, Populus y otros. Tradicionalmente había predominado ampliamente pino radiata en las plantaciones, pero desde la década de los 90 del siglo pasado empezó a incrementarse fuertemente la participación de eucaliptos, es así como en 1990 la superficie plantada con eucaliptos era de 101.700 ha y a 2015 se registran 848.869 ha, de las cuales 576.937 ha corresponden a E. globulus y 259.299 ha a $E$. nitens.

El consumo de madera en trozas por la industria forestal es en 2016 de 44.556 .000 $\mathrm{m}^{3} \mathrm{ssc}$, el cual es provisto casi exclusivamente por las plantaciones forestales (algunas especies nativas participan solo en un $0,7 \%$ ). Este consumo era en el año 2000 de $24.436,800 \mathrm{~m}^{3}$ ssc y en el año 2010 de $34.559 .600 \mathrm{~m}^{3} \mathrm{ssc}$, lo que refleja el desarrollo y constante ampliación de la industria forestal chilena.

Dada la importancia del recurso plantaciones, el Instituto Forestal (INFOR) realiza periódicamente el estudio Disponibilidad Futura de Madera de Plantaciones de Pino Radiata y Eucalipto a un horizonte de 30 años, y este estudio requiere conocer la tendencia en el uso de especies en las plantaciones para la estimación de las disponibilidades futuras de madera de una y otra. Este estudio se centra en la reforestación y en los pequeños y medianos propietarios, dado que las grandes empresas forestales contribuyen con las proyecciones de sus propias plantaciones.

El presente trabajo analiza la tendencia en el uso de las especies en las reforestaciones de pequeños y medianos propietarios revisando el período comprendido entre los años 2012 y 2016. Los resultados indican que este segmento de propietarios reforesta en el período 152.637 ha y que es el subsegmento pequeños propietarios el que domina en esta cifra con 130.982 ha. Además, es este subsegmento el que está empleando mayormente los eucaliptos, con 1,2 ha reforestada con eucaliptos por cada 1 ha reforestada con pinus radiata, en tanto que los medianos propietarios registran 0,6 ha reforesta con eucaliptos por cada 1 ha reforestada con pinus radiata.

Palabras clave: Pequeños y medianos propietarios, reforestación, tendencia en el uso de especies.

\footnotetext{
${ }^{10}$ Investigadores, Instituto Forestal, Sede Bio Bio. aavila@infor.cl
} 


\section{SUMMARY}

Total planted forest area in Chile by 2015 is 2.4 million hectares and the plantation annual rate remains over than 90 thousand hectares, however this rate is represented nowadays basically by reforestation (harvested areas replacement). Since 2012, expiry year of the afforestation state incentives law, the afforestation annual rate fell drastically. In 2016 reforestation rate was 96 thousand hectares while afforestation rate was only 2.4 thousand hectares.

Main species in Chilean planted forest are by 2015 Pinus radiata, Eucalyptus globulus and Eucalyptus nitens, reaching to 1.400 .259 ha, 576.937 ha and 259.299 ha, respectively. The difference respect to the total planted area is done by 147.434 ha of other species plantations from genus like Pinus, Pseudotsuga, Populus and others. Traditionally the main species was Pinus radiata, but since the 90's decade of the past century eucalypts plantations strongly increased; by 1990 Eucalypts plantations were 101,700 ha and by 2015 have increased to 848,869 ha, of which 576.937 are E. globulus plantations and 259,299 E. nitens plantations.

The annual forest industry round wood consumption in 2016 was 44,556,000 $\mathrm{m}^{3}$, provided almost exclusively by the planted forests (some native species participate only with $0.7 \%$ ). This consumption level was in 2000 of $24,436,800 \mathrm{~m}^{3}$ and in the year 2010 of $34,559,600 \mathrm{~m}^{3}$, figures which reflect the Chilean forestry industry development and permanent expansion.

Considering the planted forest resources importance, the Instituto Forestal (INFOR) carry out periodically the study Future Radiata Pine and Eucalypts Plantations Wood Availability, under a 30 year time frame, and this study requires the knowledge of the species use in planted forests trend in order to estimate the future wood availability by species. The study focuses on reforestation and on small and medium owners because the large forest companies provide their own plantation proyections.

The present paper analyses the small and medium owners species use trend in their reforestations, reviewing the 2012 - 2016 period. Results show that during the period small and medium owners reforest 152,637 ha, and the small owners segment dominates with 130,982 ha. Also this segment is using mainly Eucalypts, reforesting 1.2 ha with Eucalypts by 1 ha with Radiata Pine, wile medium owners reforest 0.6 ha with Eucalypts by 1 ha with Radiata Pine.

Key words: Small and medium owners, reforestation, species use trend. 


\section{INTRODUCCIÓN}

El consumo de madera en trozas de la industria forestal chilena es en 2016 de 44,6 millones de metros cúbicos y está basado fundamentalmente en las plantaciones forestales, el $69,2 \%$ de este volumen es provisto por plantaciones de pino radiata (Pinus radiata D. Don.), el $30,1 \%$ por plantaciones de eucaliptos (Eucalyptus globulus Labill. y Eucalyptus nitens $\mathrm{H}$. Deane \& Maiden), en tanto que diversas especies de los bosques nativos proveen solo el $0,7 \%$ (INFOR, 2017).

En el marco del estudio de disponibilidad futura de madera de plantaciones que realiza periódicamente el Instituto Forestal (INFOR), se definieron categorías de propietarios de este recurso en función de su patrimonio total de plantaciones:

$$
\begin{aligned}
& \text { Grandes empresas ( }>30 \text { mil ha) } \\
& \text { Empresas medianas ( }>5 \mathrm{mil}<30 \mathrm{mil} \mathrm{ha}) \\
& \text { Medianos propietarios ( }>200 \mathrm{ha}<5 \mathrm{mil} \mathrm{ha} \text { ) } \\
& \text { Pequeños propietarios }(\leq 200 \mathrm{ha})
\end{aligned}
$$

En el estudio de disponibilidad (año 2017), participan grandes empresas y algunas empresas medianas, que aportan información y proyecciones sobre su patrimonio.

En el caso de los Pequeños y Medianos Propietarios (PYMP), en el estudio anterior de disponibilidad (año 2012), se hicieron análisis sobre el comportamiento histórico en sus plantaciones, en particular lo referente al cambio de especie de pino radiata a eucaliptos.

En esta oportunidad, estudio de disponibilidad 2017, también se requiere estimar el comportamiento de este segmento de propietarios, para las tres especies principales plantadas en Chile; Pinus radiata, Eucalyptus globulus y Eucalyptus nitens, en lo referente a los cambios o reemplazos de especies en sus plantaciones, y esta vez se analiza separadamente para pequeños y para medianos propietarios, en base a las intenciones de reforestaciones presentadas a la Corporación Nacional Forestal (CONAF).

\section{MÉTODO}

Se revisaron los datos de reforestación presentadas a CONAF, entre los años 2012 y 2016; se seleccionaron y clasificaron los propietarios de acuerdo a los criterios de superficie establecidos en el estudio de disponibilidad futura de madera 2012 (INFOR, 2013), en las regiones de Valparaíso, O’Higgins, Maule, Bio Bio, Araucanía, Los Ríos y Los Lagos; no se incluyó la región Metropolitana, porque no registró superficie reforestada con pino radiata.

Se analizaron las distribuciones de superficie reforestada por especie y tipo de propietario en cada región del área de estudio.

Se utilizó el criterio de razón de reforestación (Avila y Muñoz, 2014), en donde se establecen las hectáreas plantadas con eucaliptus por cada hectárea plantada con pino radiata. En esta razón se sumaron las superficies de ambas especies del género Eucalyptus (E. globulus y $E$. nitens).

\section{RESULTADOS}

\section{Intenciones de Reforestación}

Se revisaron las solicitudes de reforestación presentadas a CONAF por pequeños propietarios y medianos propietarios, en todas las regiones del estudio, con las especies Pinus 
radiata, Eucalyptus globulus, Eucalyptus nitens, entre los años 2012 y 2016 ,

\section{- $\quad$ Intenciones de Reforestar con la Especie Pinus radiata}

Durante el período 2012-2016, los PYMP presentaron a CONAF en el área de estudio solicitudes de reforestar con esta especie por 75.182 ha, de las cuales el $81 \%$ corresponde a pequeños propietarios (PP).

Este segmento mostró en promedio una tasa de reforestación en torno a las 12 mil ha al año dentro de las regiones en estudio.

Se aprecia en la Figura $N^{\circ} 1$ una baja en el año 2015 (5.745 ha) y al año siguiente el valor más alto (16.436 ha).

Respecto de los medianos propietarios (MP), las intenciones de reforestar con pino radiata aparecen en torno a 2.800 ha en promedio, con variaciones menos marcadas que en pequeños propietarios.

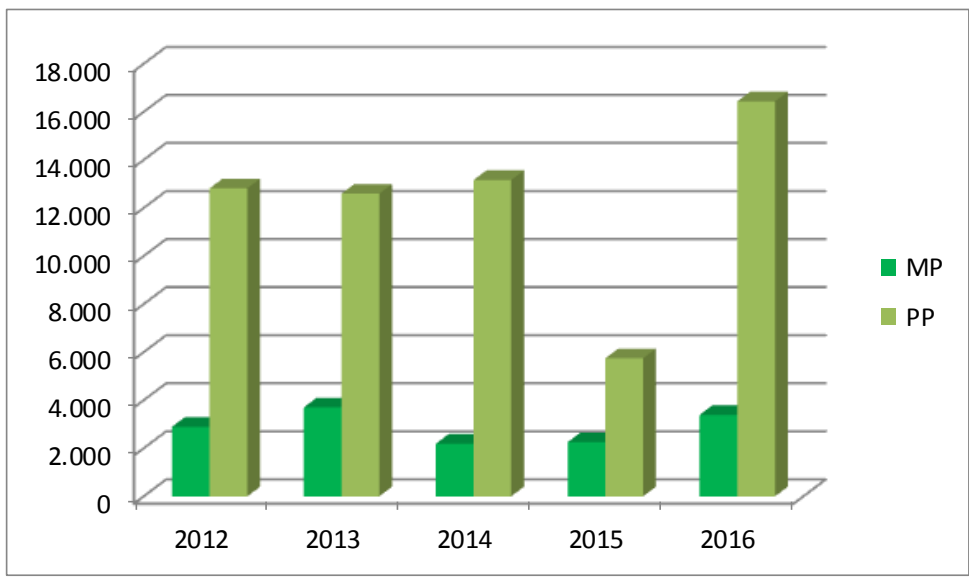

Figura $\mathrm{N}^{\circ} 1$

SUPERFICIE REFORESTACIONES Pinus radiata POR TIPO DE PROPIETARIO

\section{- Intenciones de Reforestar con la Especie Eucalyptus globulus}

Eucalyptus globulus fue la segunda especie en superficie reforestada por los PYMP, con 68.269 ha en las regiones que comprende el estudio.

También son los PP quienes concentran estas reforestaciones con $89 \%$, salvo en el año 2015, se aprecia una tendencia al incremento de superficie desde las 11 mil hectáreas en 2012 , hasta las 15.800 ha en el año 2016 (Figura $N^{\circ} 2$ ).

Los MP en tanto muestran una distribución que baja desde el año 2012 hasta 2015, para nuevamente incrementarse en torno a las 2.500 hectáreas en el año 2016. 


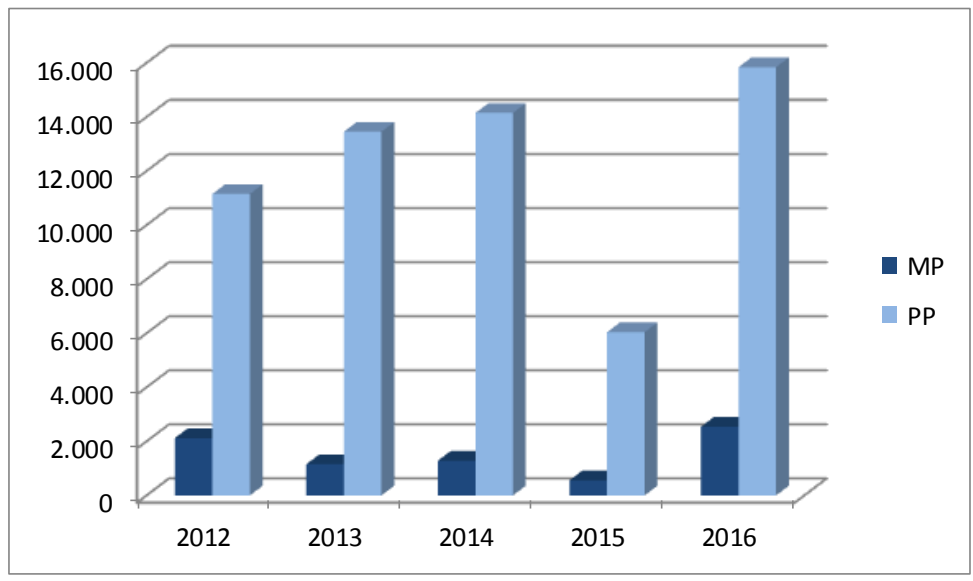

Figura $\mathbf{N}^{\circ} 2$

SUPERFICIE REFORESTACIONES Eucalyptus globulus POR TIPO DE PROPIETARIO

\section{- $\quad$ Intenciones de Reforestar con la Especie Eucalyptus nitens}

Eucalyptus nitens es la tercera en importancia en términos de superficie, pero como se observa en la Figura $\mathrm{N}^{\circ} 3$ con tasas anuales muy menores a las otras dos especies.

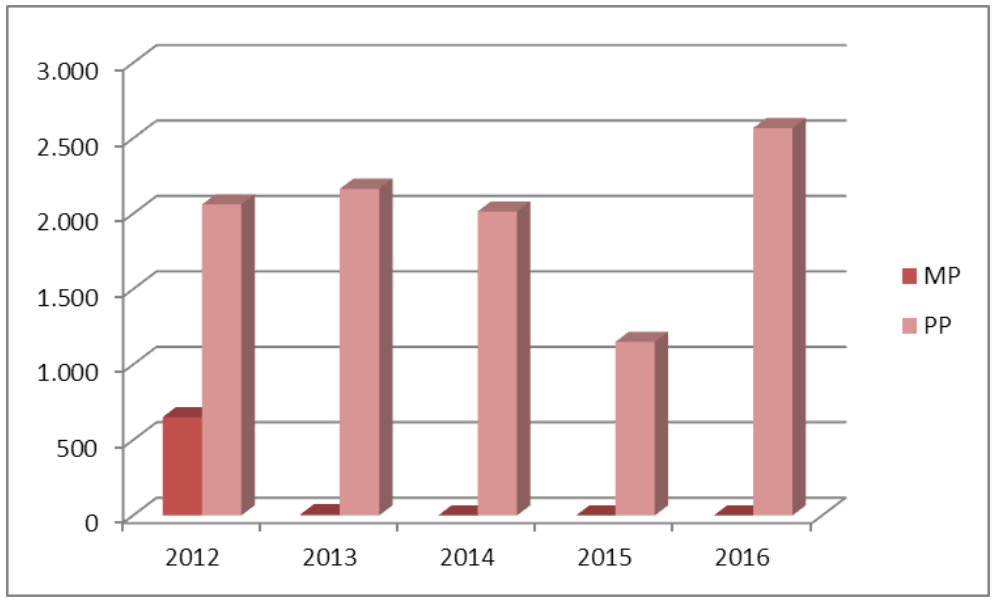

Figura $\mathrm{N}^{\circ} 3$

SUPERFICIE REFORESTACIONES Eucalyptus nitens POR TIPO DE PROPIETARIO

Los PP son el segmento que mostró la mayor superficie de reforestación con esta especie, en promedio cerca de 2.000 ha al año. Tal como ocurrió con las otras especies, el año 2015 presentó un brusco descenso, para luego incrementarse en 2.500 ha.

Los MP en tanto muestran reforestación importante con esta especie solo el año 2012 (650 ha), para luego hacerse nula esta reforestación en los últimos tres años del análisis. 


\section{Reforestaciones por Región}

A continuación, se presentan las reforestaciones con las tres especies, por cada región administrativa y tipo de propietario.

\section{Región de Valparaíso}

Eucalyptus globulus es la especie más empleada por los MP en sus reforestaciones en esta región. Como se aprecia en la Figura $N^{\circ} 4$, en promedio 408 ha para el período, pero con un máximo de 878 ha y un mínimo de 0 ha, en los años 2014 y 2015, respectivamente. Muy baja participación de Pinus radiata y no se registra superficie reforestada con Eucalyptus nitens.

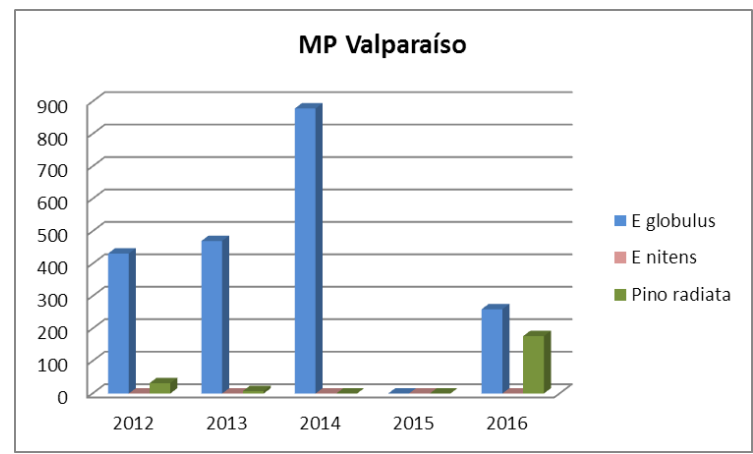

Figura $N^{\circ} 4$

REFORESTACIONES DE MP EN LA REGIÓN DE VALPARAÍSO

En cuanto a los PP, presentan un comportamiento similar en lo que respecta a la mayor reforestación con Eucalyptus globulus, menor con Pinus radiata y nula con Eucalyptus nitens; pero con mayor superficie que la mostrada por los MP (Figura $N^{\circ} 5$ ). En promedio se plantaron 911 ha anuales con Eucalyptus globulus, con un máximo de 1.557 ha en el año 2013, y un mínimo de 0 ha en 2015. Se aprecia una tendencia a la disminución de la superficie reforestada. Los PP reforestaron en promedio 76 ha con Pinus radiata, alcanzando en 2016 la mayor superficie con 144 ha.

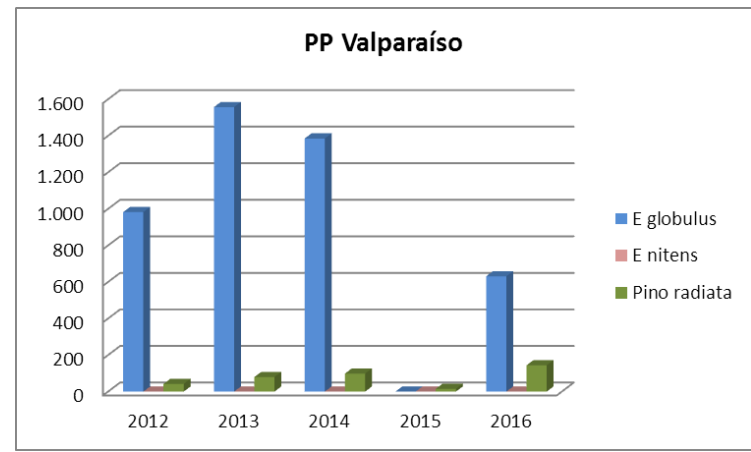

Figura $N^{\circ} 5$

REFORESTACIONES DE PP EN LA REGIÓN DE VALPARAÍSO 


\section{- $\quad$ Región de O’Higgins}

En la región de O'Higgins los MP reforestaron mayoritariamente con la especie Pinus radiata (Figura $N^{\circ} 6$ ), 512 ha en promedio, presentando como máximo 800 ha en el año 2012, solo 83 ha en promedio anual se reforestó con Eucalyptus globulus y con clara tendencia a la baja. propietarios.

No se registran reforestaciones con Eucalyptus nitens, por parte de este segmento de

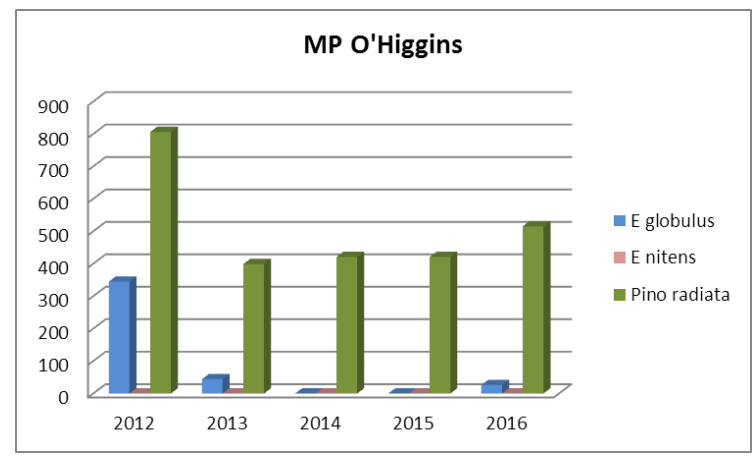

Figura $N^{\circ} 6$

REFORESTACIONES DE MP EN LA REGIÓN DE O'HIGGINS

Los PP en cambio, reforestaron mayoritariamente con Eucalyptus globulus (Figura № 7), con un promedio para el período de 1.401 ha, alcanzando el máximo en año 2014 con 2.020 ha, para declinar en los dos años siguientes.

Con Pinus radiata se reforestó en promedio anual 885 ha, con menos variaciones entre los años que lo observado en Eucalyptus globulus.

No se reforestó con Eucalyptus nitens, por parte de este tipo de propietario.

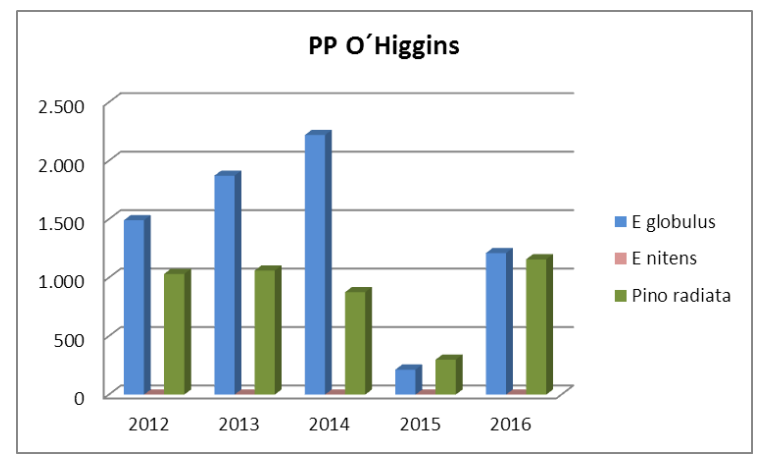

Figura $\mathrm{N}^{\circ} 7$

REFORESTACIONES DE PP EN LA REGIÓN DE O'HIGGINS 


\section{- Región del Maule}

Los MP de la región del Maule reforestaron mayoritariamente con Pinus radiata, en promedio 1.070 ha por año (Figura $N^{\circ} 8$ ).

Le sigue Eucalyptus globulus que muestra un promedio de 111 ha/año, pero con una marcada disminución desde el año 2012.

No aparecieron superficies reforestadas con Eucalyptus nitens por parte de los MP.

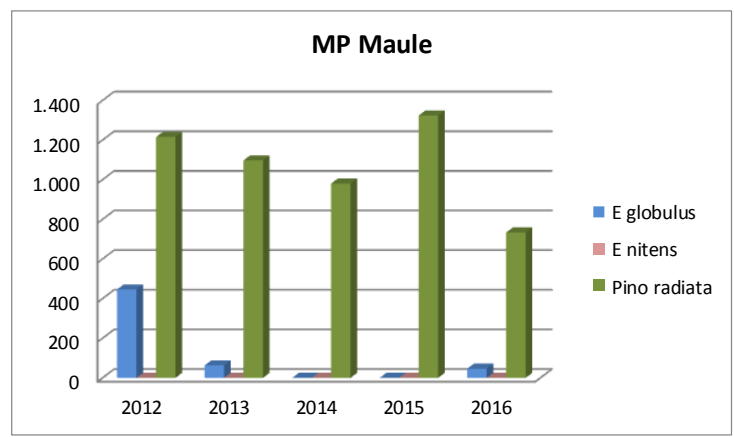

Figura $N^{\circ} 8$

REFORESTACIONES DE MP EN LA REGIÓN DEL MAULE

Los PP del Maule quintuplicaron la superficie reforestada por los MP. También reforestaron principalmente con Pinus radiata (Figura $\mathrm{N}^{\circ}$ 9), con 5.394 ha/año en promedio, destacando como máximo el año 2016 con 7.470 ha.

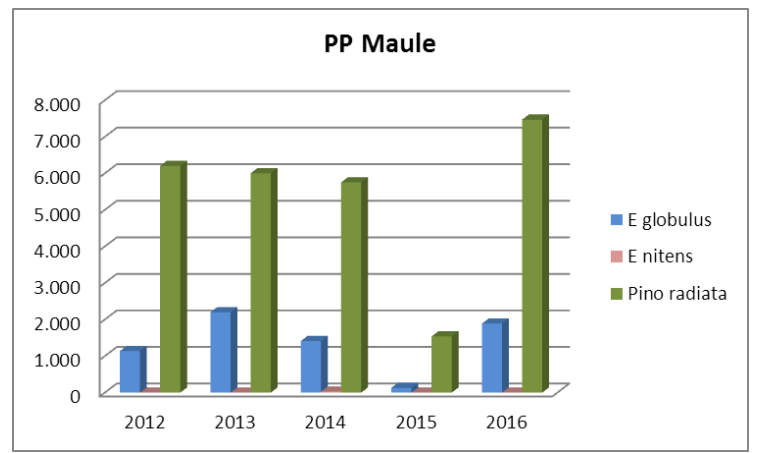

Figura $N^{\circ} 9$

REFORESTACIONES DE PP EN LA REGIÓN DEL MAULE

Durante el período analizado, Eucalyptus globulus registra un promedio de 1.354 ha/año de reforestación por parte de los PP.

Con Eucalyptus nitens se reforestaron solamente 30 ha en los cinco años. 


\section{- Región del Bio Bio}

La mayor superficie reforestada por los MP del Bio Bio correspondió a la especie Pinus radiata, en promedio 737 ha/año; alcanzando el máximo en el año 2013 con 1.155 ha (Figura Nº 10).

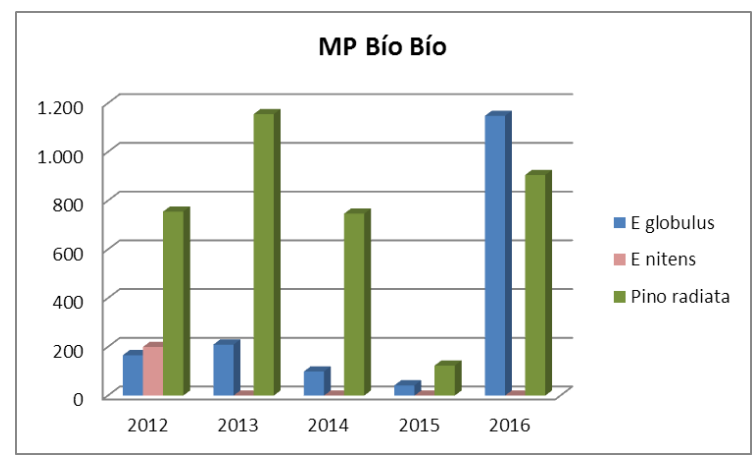

Figura 10

REFORESTACIONES DE MP EN LA REGIÓN DEL BIO BIO

Con Eucalyptus globulus se aprecia una baja superficie reforestada por parte de los MP, sin embargo, el año 2016 superó a la superficie reforestada con pino radiata.

Solo se aprecia Eucalyptus nitens plantado en reforestaciones durante el año 2012.

Los PP en tanto, reforestaron superficies ocho veces superiores a la de los MP. La situación varía en este segmento porque Eucalyptus globulus fue la especie más plantada (Figura $\left.\mathrm{N}^{\circ} 11\right)$, con 4.683 ha/año en promedio y un máximo en 2016 de 6.948 ha.

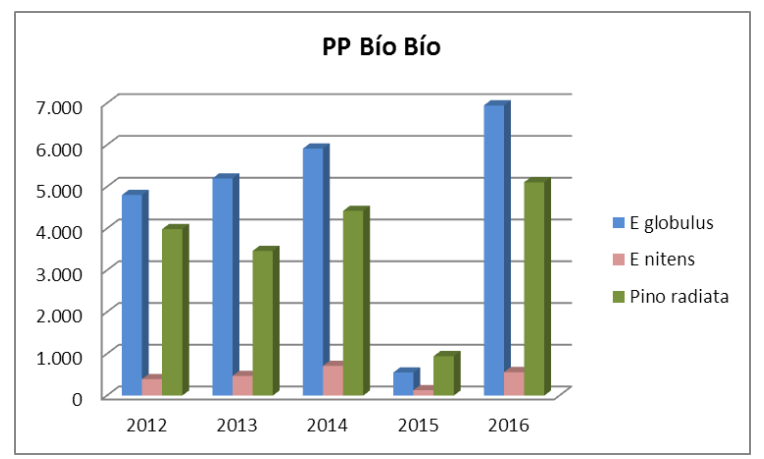

Figura $\mathrm{N}^{\circ} 11$

REFORESTACIONES DE PP EN LA REGIÓN DEL BIO BIO

Los PP del Bio Bio reforestaron en promedio 3.585 ha/año con Pinus radiata y 455 ha/año con Eucalyptus nitens. 


\section{- Región de la Araucanía}

En la región de la Araucanía los MP reforestaron en promedio 487 ha con Eucalyptus globulus, con un máximo en 2016 de 1.059 ha (Figura $N^{\circ}$ 12), superando levemente a pino radiata que registra 368 ha/año en promedio), que también alcanzó su máximo el año 2016.

Eucalyptus nitens aparece solo a comienzo del período, durante el año 2012.

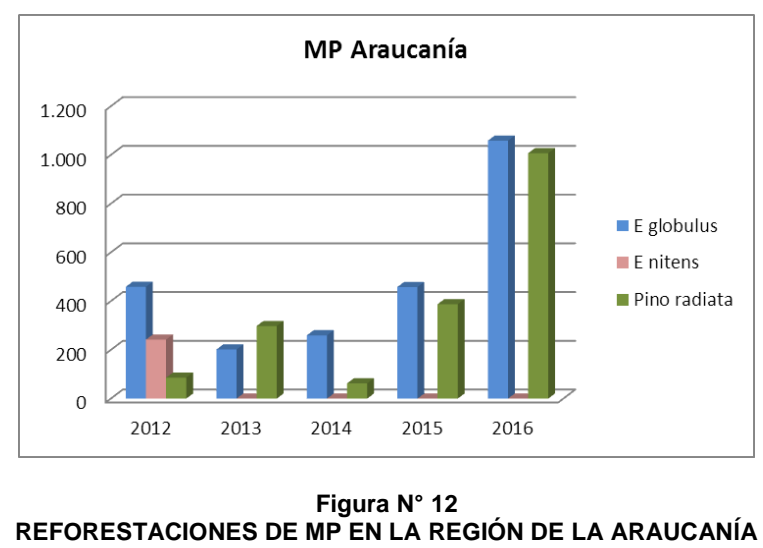

Eucalyptus globulus fue la especie con mayor superficie reforestada por los PP en esta región, 3.328 ha en promedio, en el año 2015 alcanzó su máximo con 4.957 ha (Figura № 13).

Pino radiata fue la segunda especie más utilizada en las reforestaciones de los $\mathrm{PP}$, con 1.867 ha/año en promedio y también alcanzó su máximo en el año 2015, con 2.695 ha.

Eucalyptus nitens, en tanto presentó superficie plantada durante todo el período, con 793 ha/año en promedio.

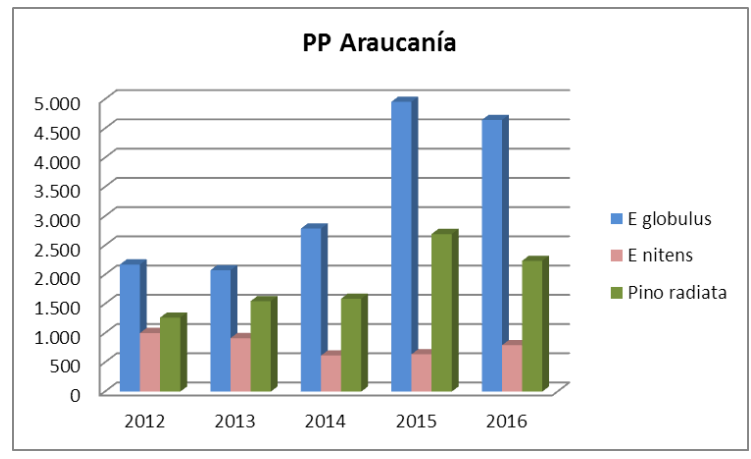

Figura $N^{\circ} 13$

REFORESTACIONES DE PP EN LA REGIÓN DE LA ARAUCANÍA 


\section{- Región de los Ríos}

Los MP de la región de los Ríos mostraron casi nula actividad en los cinco años.

Se registran en el período reforestaciones de solo 7 ha de Eucalyptus nitens y 6 ha de pino radiata (Figura $\mathrm{N}^{\circ} 14$ ).

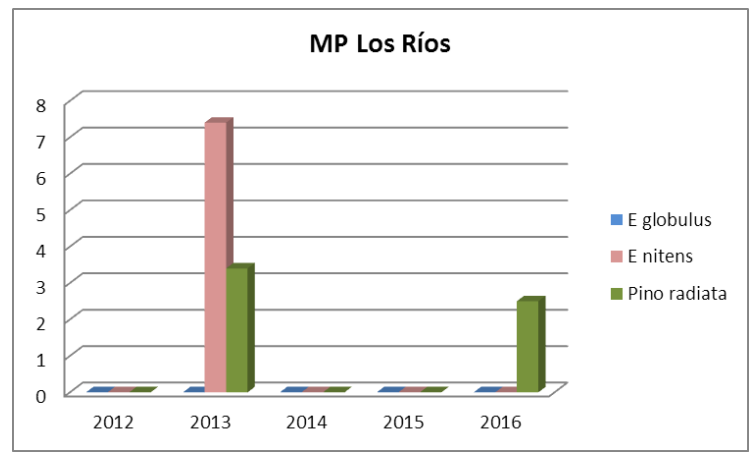

Figura $\mathrm{N}^{\circ} 14$

REFORESTACIONES DE MP EN LA REGIÓN DE LOS RÍOS

Eucalyptus nitens fue la especie con mayor superficie reforestada por los PP en esta región (Figura $\mathrm{N}^{\circ} 15$ ), con 487 ha/año en promedio. promedio.

Le siguen Eucalyptus globulus con 234 ha/año y pino radiata con 202 ha/año en

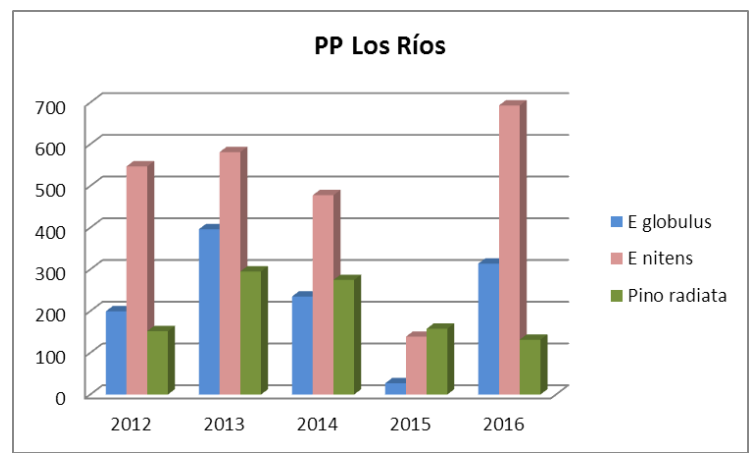

Figura $\mathrm{N}^{\circ} 15$

REFORESTACIONES DE PP EN LA REGIÓN DE LOS RÍOS 


\section{- $\quad$ Región de los Lagos}

Los MP de la región de los Lagos mostraron un comportamiento irregular, dado que la mayor especie reforestada fue Eucalyptus nitens con 210 ha en total, pero solo se registró en el año 2012 (Figura N 16).

Le siguió Eucalyptus globulus con 172 ha en total plantadas en dos años.

Finalmente, pino radiata con 48 ha al final del período.

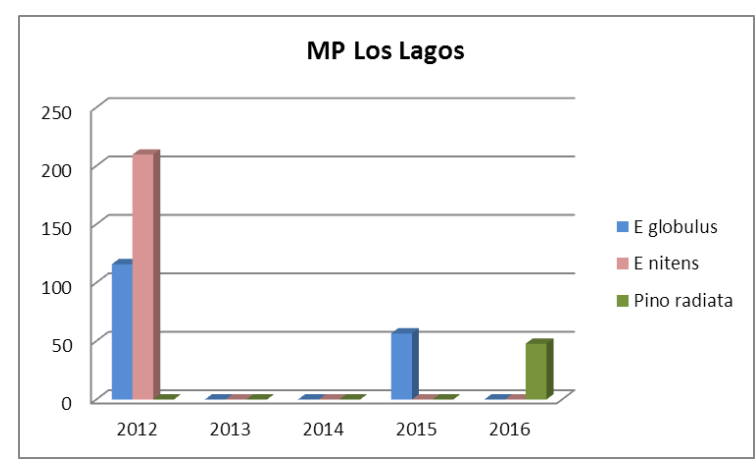

Figura $N^{\circ} 16$

REFORESTACIONES DE MP EN LA REGIÓN DE LOS LAGOS

Por su parte los PP en la región los Lagos reforestaron principalmente con Eucalyptus nitens (Figura N 17), 251 ha/año en promedio y un máximo de 518 ha en el año 2016. Le siguen Pino radiata y Eucalyptus globulus, con 143 y 142 ha en promedio,
respectivamente. Le siguen Pino radiata y Eucalyptus globulus, con 143 y 142 ha en promedio,
respectivamente.

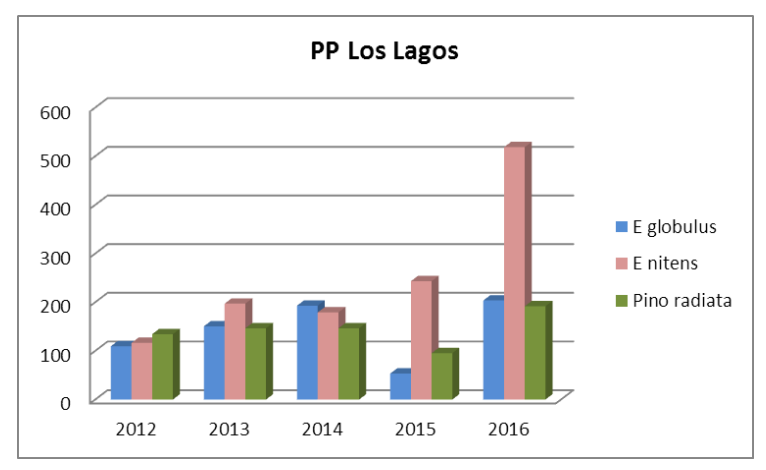

Figura $N^{\circ} 17$

REFORESTACIONES DE PP EN LA REGIÓN DE LOS LAGOS 


\section{Razón de Reforestación}

A continuación se presentan los resultados, para cada región y tipo de propietario, de la razón de reforestación $(\mathrm{RR})$, es decir, la relación de superficie reforestada con eucaliptos por cada hectárea reforestada con pino radiata. En este caso se unen las superficies de ambas especies del género Eucalyptus en estudio.

\section{- Región de Valparaíso}

En la Figura $\mathrm{N}^{\circ} 18$ se presenta la RR para los dos tipos de propietarios; los MP mostraron una alta razón los años 2012 y 2013 (13 y 61, respectivamente), para bajar fuertemente los tres años siguientes debido a la nula o baja superficie reforestada con pino radiata. Los PP presentaron una razón de 23 en 2012 para ir declinando en los siguientes años. El año 2015 no se registraron superficies reforestadas con eucaliptos.

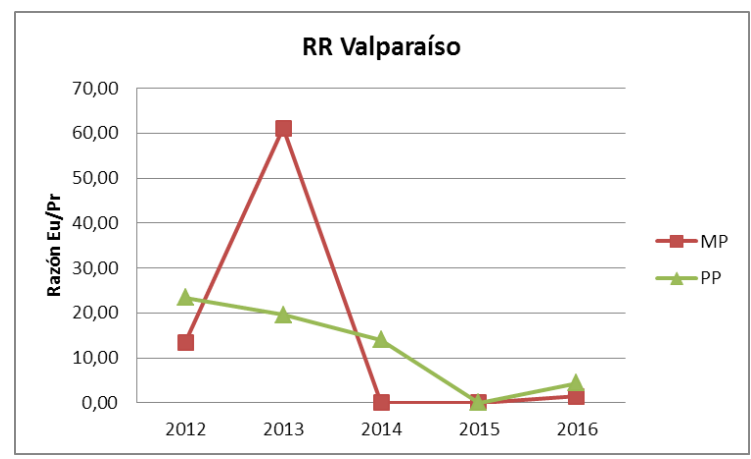

Figura $N^{\circ} 18$

RAZÓN DE REFORESTACIÓN POR TIPO DE PROPIETARIO REGIÓN DE VALPARAÍSO

\section{- Región de O’Higgins}

Los MP exhibieron una RR menor a 1 en todo el período (Figura $N^{\circ} 19$ ) y con tendencia a la baja. En tanto los PP presentaron RR que creció de 1,4 en 2012 hasta 2,5 máximo en el año 2014, para descender en los dos años siguientes en donde las superficies de eucaliptos y pino radiata fueron similares.

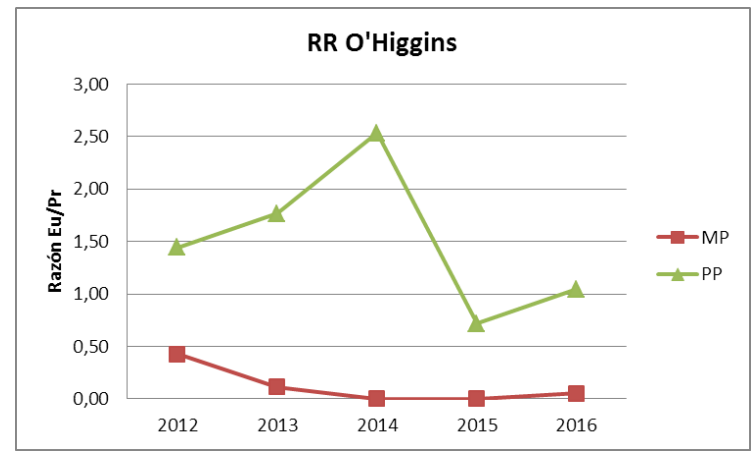

Figura $N^{\circ} 19$

RAZÓN DE REFORESTACIÓN POR TIPO DE PROPIETARIO REGIÓN DE O’HIGGINS 


\section{- Región del Maule}

Durante todo el período, los MP del Maule presentaron RR menores a 1 (Figura $N^{\circ} 20$ ), incluso en los años 2014 y 2015 no hubo superficie reforestada con eucaliptos.

También los PP del Maule exhibieron RR inferiores a 1 durante los 5 años, con variaciones entre los años.

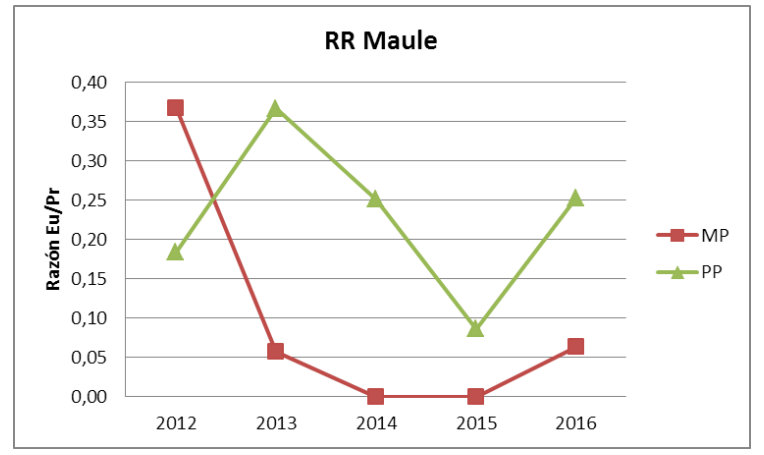

Figura $N^{\circ} 20$

RAZÓN DE REFORESTACIÓN POR TIPO DE PROPIETARIO REGIÓN DEL MAULE

\section{- Región del Bio Bio}

Los MP de esta región mostraron RR inferiores a 1 los cuatro primeros años (Figura $\mathrm{N}^{\circ}$ 21), solo el año 2016 alcanzó razón 1,3.

Los PP del Bio Bio muestran RR en torno a 1,5, excepto el año 2015 que es inferior a 1.

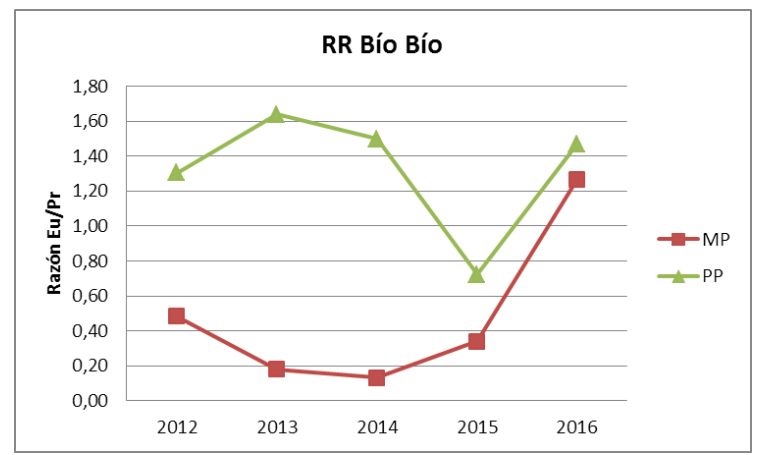

Figura $\mathrm{N}^{\circ} 21$

RAZÓN DE REFORESTACIÓN POR TIPO DE PROPIETARIO REGIÓN DEL BIO BIO 


\section{- Región de la Araucanía}

El año 2012 los MP de la Araucanía presentaron RR sobre 8, para descender de 1 al año siguiente, en 2014 nuevamente se incrementa a 4 (Figura $N^{\circ} 22$ ) y los dos últimos años se mantiene alrededor de 1.

Los PP de la Araucanía en cambio, se mostraron más estables con razones en torno a 2.

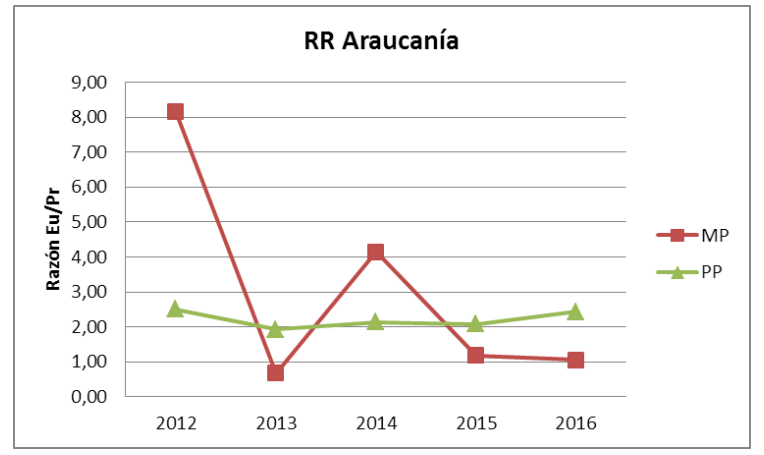

Figura $N^{\circ} 22$

RAZÓN DE REFORESTACIÓN POR TIPO DE PROPIETARIO REGIÓN DE LA ARAUCANÍA

\section{- Región de los Ríos}

Como se indicó anteriormente, los MP de Los Ríos mostraron muy baja superficie reforestada de las tres especies, por lo que varios años la RR tuvo valores indefinidos (división por cero). Esta situación se aprecia en la Figura № 23.

En tanto los PP presentaron razones de 5 en el año 2012, con tendencia descendente hasta el año 2015, para alcanzar el máximo el 2016 (RR 7,67).

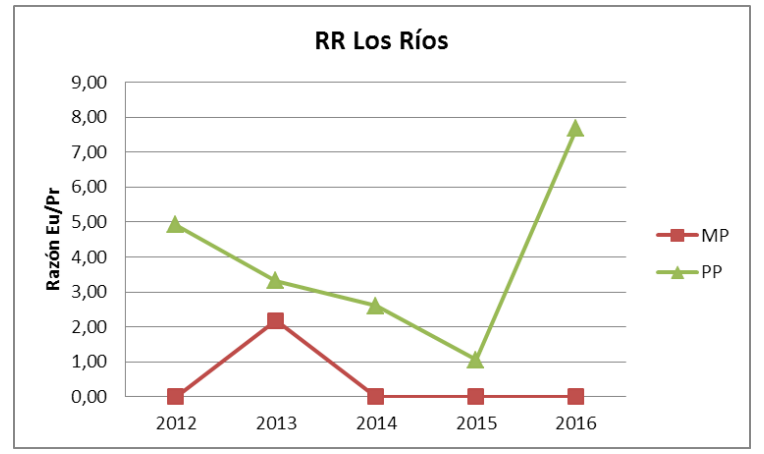

Figura $N^{\circ} 23$

RAZÓN DE REFORESTACIÓN POR TIPO DE PROPIETARIO REGIÓN DE LOS RÍOS 


\section{- $\quad$ Región de Los Lagos}

La nula reforestación con pino radiata por parte de los MP los cuatro primeros años y con eucaliptos en algunos años incide en que la RR muestre valores indefinidos o cero (Figura $N^{\circ} 24$ ).

Los PP de la región exhibieron una razón con tendencia al alza durante todo el período de análisis, con RR 1,7 el año 2012 hasta alcanzar el máximo 3,8 en el año 2016.

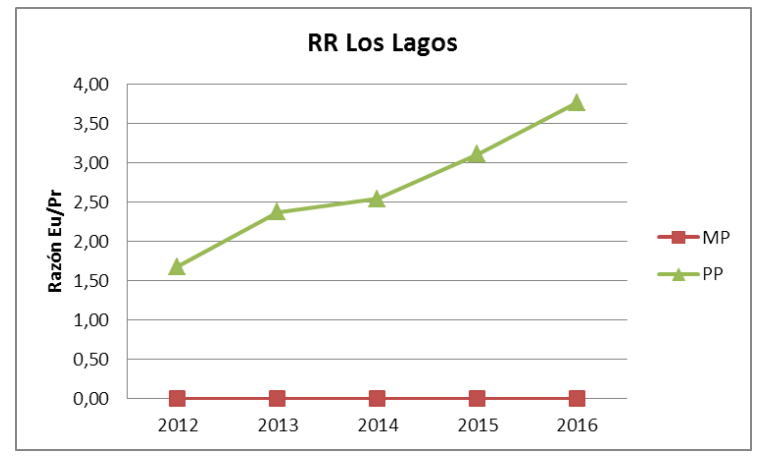

Figura $N^{\circ} 24$

RAZÓN DE REFORESTACIÓN POR TIPO DE PROPIETARIO REGIÓN DE LOS LAGOS

\section{CONCLUSIONES}

En todas las regiones administrativas del área de estudio el mayor aporte en superficie de reforestación correspondió al segmento de los pequeños propietarios, con el $81 \%$ de la superficie reforestada con pino radiata, el $89 \%$ de la reforestada con Eucalyptus globulus y el $94 \%$ de aquella reforestada con Eucalyptus nitens.

Las superficies reforestadas con estas especies variaron a través de las regiones, siendo Bio Bio con 49.000 ha, Maule con 39.000 ha y Araucanía con 34.000 ha las regiones con mayor superficie reforestada por pequeños y medianos propietarios durante el período 2012-2016.

También existieron variaciones en cuanto a la principal especie plantada; Eucalyptus globulus fue la principal especie en las regiones de Valparaíso y Araucanía; pino radiata en la región del Maule y Eucalyptus nitens en las regiones de Los Ríos y Los Lagos. En las regiones de O'Higgins y Bio Bio la principal especie plantada por los pequeños propietarios fue Eucalyptus globulus, en tanto que para los medianos propietarios fue pino radiata.

En cuanto a la razón de reforestación, también existieron diferencias entre regiones y en algunos casos, entre tipo de propietarios. Los cocientes más altos se encontraron en la región de Valparaíso, tanto en medianos como en pequeños propietarios, con 61 y 23 ha reforestadas, respectivamente, con eucaliptos por cada hectárea reforestada con pino radiata, aunque en medianos propietarios el cociente cae por falta de superficie reforestada con pino radiata en los años 2014 y 2015.

En las regiones de Los Ríos, Los Lagos y Araucanía las razones de reforestaciones también son superiores a 1 para los pequeños propietarios.

En las regiones de O'Higgins y Bio Bio en tanto es superior a 1 para pequeños propietarios e inferior a 1 en medianos propietarios.

En las regiones de Los Ríos y Los Lagos los medianos propietarios presentaron varios 
años sin superficies reforestadas con pino radiata (cociente indefinido).

Finalmente, en la región del Maule, tanto medianos como pequeños propietarios presentaron razones de reforestaciones menores que 1 , se reforestaron en promedio 0,1 ha y 0,23 ha con eucaliptos, respectivamente, por cada hectárea reforestada con pino radiata.

\section{REFERENCIAS}

INFOR. 2017. Anuario Forestal 2017. Boletín Estadístico № 159. Instituto Forestal, Chile. 175 p.

INFOR. 2013. Disponibilidad de Madera de Plantaciones de Pino Radiata y Eucalipto (2010-2040). Informe Técnico $N^{\circ} 194$. Instituto Forestal, Chile. 115 p.

Avila, A, y Muñoz, J. C., 2014. Tendencias de Cambio de Especies Forestales en Plantaciones de Pequeños y Medianos Propietarios. Publicado en: E: Barros, S. (Ed.) Ciencia e Investigación Forestal, Vol. 20 № 3 . Instituto Forestal, Chile. Pp: 53-70. 
APÉNDICE № 1

SUPERFICIE REFORESTADA POR REGIÓN, ESPECIE Y TIPO DE PROPIETARIO

Región de Valparaíso Mediano Propietario

\begin{tabular}{|c|r|r|r|r|}
\hline \multirow{2}{*}{ Año } & E. globulus & \multicolumn{1}{|c|}{ E. nitens } & \multicolumn{1}{c|}{ Pino radiata } & \multicolumn{1}{c|}{ Total } \\
\cline { 2 - 5 } & \multicolumn{5}{|c|}{ ( ha ) } \\
\hline 2012 & 431,5 & 0,0 & 32,0 & 463,5 \\
\hline 2013 & 470,3 & 0,0 & 7,7 & 478,0 \\
\hline 2014 & 878,5 & 0,0 & 0,0 & 878,5 \\
\hline 2015 & 0,0 & 0,0 & 0,0 & 0,0 \\
\hline 2016 & 259,7 & 0,0 & 176,8 & 436,5 \\
\hline Total & $\mathbf{2 . 0 3 9 , 9}$ & $\mathbf{0 , 0}$ & $\mathbf{2 1 6 , 5}$ & $\mathbf{2 . 2 5 6 , 4}$ \\
\hline Promedio & $\mathbf{4 0 8 , 0}$ & $\mathbf{0 , 0}$ & $\mathbf{4 3 , 3}$ & $\mathbf{4 5 1 , 3}$ \\
\hline
\end{tabular}

Región de Valparaíso Pequeño Propietario

\begin{tabular}{|c|r|r|r|r|}
\hline \multirow{2}{*}{ Año } & E. globulus & \multicolumn{1}{|c|}{ E. nitens } & \multicolumn{1}{c|}{ Pino radiata } & \multicolumn{1}{c|}{ Total } \\
\cline { 2 - 5 } & \multicolumn{5}{|c|}{ ( ha ) } \\
\hline 2012 & 982,3 & 0,0 & 41,9 & $1.024,2$ \\
\hline 2013 & $1.556,8$ & 0,0 & 79,5 & $1.636,3$ \\
\hline 2014 & $1.385,1$ & 0,0 & 98,8 & $1.483,9$ \\
\hline 2015 & 0,0 & 0,0 & 15,2 & 15,2 \\
\hline 2016 & 631,0 & 0,0 & 143,8 & 774,8 \\
\hline Total & $\mathbf{4 . 5 5 5 , 2}$ & $\mathbf{0 , 0}$ & $\mathbf{3 7 9 , 2}$ & $\mathbf{4 . 9 3 4 , 4}$ \\
\hline Promedio & $\mathbf{9 1 1 , 0}$ & $\mathbf{0 , 0}$ & $\mathbf{7 5 , 8}$ & $\mathbf{9 8 6 , 9}$ \\
\hline
\end{tabular}

Región de O’Higgins Mediano Propietario

\begin{tabular}{|c|r|r|r|r|}
\hline \multirow{2}{*}{ Año } & E. globulus & \multicolumn{1}{|c|}{ E. nitens } & \multicolumn{1}{c|}{ Pino radiata } & \multicolumn{1}{c|}{ Total } \\
\cline { 2 - 5 } & \multicolumn{5}{|c|}{ ( ha ) } \\
\hline 2012 & 344,7 & 0,0 & 806,2 & $1.150,9$ \\
\hline 2013 & 44,2 & 0,0 & 399,0 & 443,2 \\
\hline 2014 & 0,0 & 0,0 & 420,7 & 420,7 \\
\hline 2015 & 0,0 & 0,0 & 420,7 & 420,7 \\
\hline 2016 & 26,1 & 0,0 & 515,6 & 541,6 \\
\hline Total & $\mathbf{4 1 5 , 0}$ & $\mathbf{0 , 0}$ & $\mathbf{2 . 5 6 2 , 2}$ & $\mathbf{2 . 9 7 7 , \mathbf { 1 }}$ \\
\hline Promedio & $\mathbf{8 3 , 0}$ & $\mathbf{0 , 0}$ & $\mathbf{5 1 2 , 4}$ & $\mathbf{5 9 5 , 4}$ \\
\hline
\end{tabular}


Región de O'Higgins Pequeño Propietario

\begin{tabular}{|c|r|r|r|r|}
\hline \multirow{2}{*}{ Año } & E. globulus & \multicolumn{1}{|c|}{ E, nitens } & \multicolumn{1}{c|}{ Pino radiata } & \multicolumn{1}{c|}{ Total } \\
\cline { 2 - 5 } & \multicolumn{4}{|c|}{ ( ha ) } \\
\hline 2012 & $1.490,9$ & 0,0 & $1.031,9$ & $2.522,9$ \\
\hline 2013 & $1.871,8$ & 0,0 & $1.060,2$ & $2.932,0$ \\
\hline 2014 & $2.219,7$ & 0,0 & 876,0 & $3.095,6$ \\
\hline 2015 & 213,7 & 0,0 & 298,8 & 512,5 \\
\hline 2016 & $1.209,6$ & 0,0 & $1.156,6$ & $2.366,1$ \\
\hline Total & $\mathbf{7 . 0 0 5 , 8}$ & $\mathbf{0 , 0}$ & $\mathbf{4 . 4 2 3 , 4}$ & $\mathbf{1 1 . 4 2 9 , 2}$ \\
\hline Promedio & $\mathbf{1 . 4 0 1 , 2}$ & $\mathbf{0 , 0}$ & $\mathbf{8 8 4 , 7}$ & $\mathbf{2 . 2 8 5 , 8}$ \\
\hline
\end{tabular}

Región del Maule Mediano Propietario

\begin{tabular}{|c|r|r|r|r|}
\hline \multirow{2}{*}{ Año } & E. globulus & \multicolumn{1}{c|}{ E. nitens } & \multicolumn{1}{c|}{ Pino radiata } & \multicolumn{1}{c|}{ Total } \\
\cline { 2 - 5 } & \multicolumn{4}{|c|}{ ( ha ) } \\
\hline 2012 & 446,7 & 0,0 & $1.215,2$ & $1.661,8$ \\
\hline 2013 & 62,9 & 0,0 & $1.097,8$ & $1.160,7$ \\
\hline 2014 & 0,0 & 0,0 & 980,5 & 980,5 \\
\hline 2015 & 0,0 & 0,0 & $1.323,9$ & $1.323,9$ \\
\hline 2016 & 46,8 & 0,0 & 733,7 & 780,5 \\
\hline Total & $\mathbf{5 5 6 , 4}$ & $\mathbf{0 , 0}$ & $\mathbf{5 . 3 5 1 , 1}$ & $\mathbf{5 . 9 0 7 , 5}$ \\
\hline Promedio & $\mathbf{1 1 1 , 3}$ & $\mathbf{0 , 0}$ & $\mathbf{1 . 0 7 0 , 2}$ & $\mathbf{1 . 1 8 1 , 5}$ \\
\hline
\end{tabular}

Región del Maule Pequeño Propietario

\begin{tabular}{|c|r|r|r|r|}
\hline \multirow{2}{*}{ Año } & E. globulus & \multicolumn{1}{|c|}{ E. nitens } & \multicolumn{1}{c|}{ Pino radiata } & \multicolumn{1}{c|}{ Total } \\
\cline { 2 - 5 } & \multicolumn{4}{|c|}{ ( ha ) } \\
\hline 2012 & $1.138,7$ & 0,0 & $6.205,2$ & $7.343,8$ \\
\hline 2013 & $2.198,3$ & 0,0 & $6.001,5$ & $8.199,8$ \\
\hline 2014 & $1.413,3$ & 29,7 & $5.752,9$ & $7.195,9$ \\
\hline 2015 & 132,6 & 0,0 & $1.538,5$ & $1.671,1$ \\
\hline 2016 & $1.887,5$ & 0,0 & $7.469,9$ & $9.357,4$ \\
\hline Total & $\mathbf{6 . 7 7 0 , 4}$ & $\mathbf{2 9 , 7}$ & $\mathbf{2 6 . 9 6 8 , 0}$ & $\mathbf{3 3 . 7 6 8 , 1}$ \\
\hline Promedio & $\mathbf{1 . 3 5 4 , 1}$ & $\mathbf{5 , 9}$ & $\mathbf{5 . 3 9 3 , 6}$ & $\mathbf{6 . 7 5 3 , 6}$ \\
\hline
\end{tabular}


Región del Bio Bio Mediano Propietario

\begin{tabular}{|c|r|r|r|r|}
\hline \multirow{2}{*}{ Año } & E. globulus & \multicolumn{1}{|c|}{ E. nitens } & \multicolumn{1}{c|}{ Pino radiata } & \multicolumn{1}{c|}{ Total } \\
\cline { 2 - 5 } & \multicolumn{4}{|c|}{ ( ha ) } \\
\hline 2012 & 166,1 & 199,9 & 755,3 & $1.121,3$ \\
\hline 2013 & 209,4 & 0,0 & $1.155,0$ & $1.364,5$ \\
\hline 2014 & 99,0 & 0,0 & 746,7 & 845,7 \\
\hline 2015 & 42,1 & 0,0 & 123,3 & 165,4 \\
\hline 2016 & $1.147,9$ & 0,0 & 905,3 & $2.053,3$ \\
\hline Total & $\mathbf{1 . 6 6 4 , 6}$ & $\mathbf{1 9 9 , 9}$ & $\mathbf{3 . 6 8 5 , 7}$ & $\mathbf{5 . 5 5 0 , 2}$ \\
\hline Promedio & $\mathbf{3 3 2 , 9}$ & $\mathbf{4 0 , 0}$ & $\mathbf{7 3 7 , 1}$ & $\mathbf{1 . 1 1 0 , 0}$ \\
\hline
\end{tabular}

Región del Bio Bio Pequeño Propietario

\begin{tabular}{|c|r|r|r|r|}
\hline \multirow{2}{*}{ Año } & E. globulus & \multicolumn{1}{c|}{ E. nitens } & \multicolumn{1}{c|}{ Pino radiata } & \multicolumn{1}{c|}{ Total } \\
\cline { 2 - 5 } & \multicolumn{4}{|c|}{ ( ha ) } \\
\hline 2012 & $4.802,5$ & 396,1 & $3.985,7$ & $9.184,3$ \\
\hline 2013 & $5.199,6$ & 472,4 & $3.464,3$ & $9.136,3$ \\
\hline 2014 & $5.913,8$ & 711,5 & $4.422,9$ & $11.048,2$ \\
\hline 2015 & 553,2 & 130,1 & 944,2 & $1.627,6$ \\
\hline 2016 & $6.947,8$ & 563,1 & $5.106,9$ & $12.617,8$ \\
\hline Total & $\mathbf{2 3 . 4 1 7 , 0}$ & $\mathbf{2 . 2 7 3 , 3}$ & $\mathbf{1 7 . 9 2 3 , 9}$ & $\mathbf{4 3 . 6 1 4 , 2}$ \\
\hline Promedio & $\mathbf{4 . 6 8 3 , 4}$ & $\mathbf{4 5 4 , 7}$ & $\mathbf{3 . 5 8 4 , 8}$ & $\mathbf{8 . 7 2 2 , 8}$ \\
\hline
\end{tabular}

Región de la Araucanía Mediano Propietario

\begin{tabular}{|c|r|r|r|r|}
\hline \multirow{2}{*}{ Año } & E globulus & \multicolumn{1}{c|}{ E nitens } & \multicolumn{1}{c|}{ Pino radiata } & \multicolumn{1}{c|}{ Total } \\
\cline { 2 - 5 } & \multicolumn{4}{|c|}{ ( ha ) } \\
\hline 2012 & 459,0 & 242,2 & 86,0 & 787,2 \\
\hline 2013 & 201,4 & 0,0 & 297,9 & 499,3 \\
\hline 2014 & 259,8 & 0,0 & 62,6 & 322,3 \\
\hline 2015 & 458,1 & 0,0 & 387,6 & 845,7 \\
\hline 2016 & $1.058,6$ & 0,0 & $1.007,0$ & $2.065,6$ \\
\hline Total & $\mathbf{2 . 4 3 6 , 9}$ & $\mathbf{2 4 2 , 2}$ & $\mathbf{1 . 8 4 1 , 0}$ & $\mathbf{4 . 5 2 0 , 1}$ \\
\hline Promedio & $\mathbf{4 8 7 , 4}$ & $\mathbf{4 8 , 4}$ & $\mathbf{3 6 8 , 2}$ & $\mathbf{9 0 4 , 0}$ \\
\hline
\end{tabular}


Región de la Araucanía Pequeño Propietario

\begin{tabular}{|c|r|r|r|r|}
\hline \multirow{2}{*}{ Año } & E. globulus & \multicolumn{1}{c|}{ E. nitens } & \multicolumn{1}{c|}{ Pino radiata } & \multicolumn{1}{c|}{ Total } \\
\cline { 2 - 5 } & \multicolumn{4}{|c|}{ ( ha ) } \\
\hline 2012 & $2.174,4$ & $1.002,6$ & $1.266,2$ & $4.443,2$ \\
\hline 2013 & $2.075,4$ & 914,4 & $1.547,2$ & $4.536,9$ \\
\hline 2014 & $2.788,3$ & 616,9 & $1.588,9$ & $4.994,1$ \\
\hline 2015 & $4.956,9$ & 638,8 & $2.695,1$ & $8.290,8$ \\
\hline 2016 & $4.645,5$ & 793,2 & $2.235,8$ & $7.674,5$ \\
\hline Total & $\mathbf{1 6 . 6 4 0 , 5}$ & $\mathbf{3 . 9 6 5 , 7}$ & $\mathbf{9 . 3 3 3 , 3}$ & $\mathbf{2 9 . 9 3 9 , 5}$ \\
\hline Promedio & $\mathbf{3 . 3 2 8 , 1}$ & $\mathbf{7 9 3 , 1}$ & $\mathbf{1 . 8 6 6 , 7}$ & $\mathbf{5 . 9 8 7 , 9}$ \\
\hline
\end{tabular}

Región de los Ríos Mediano Propietario

\begin{tabular}{|c|r|r|r|r|}
\hline \multirow{2}{*}{ Año } & E. globulus & \multicolumn{1}{|c|}{ E. nitens } & \multicolumn{1}{c|}{ Pino radiata } & \multicolumn{1}{c|}{ Total } \\
\cline { 2 - 5 } & \multicolumn{5}{|c|}{ ( ha ) } \\
\hline 2012 & 0,0 & 0,0 & 0,0 & 0,0 \\
\hline 2013 & 0,0 & 7,4 & 3,4 & 10,8 \\
\hline 2014 & 0,0 & 0,0 & 0,0 & 0,0 \\
\hline 2015 & 0,0 & 0,0 & 0,0 & 0,0 \\
\hline 2016 & 0,0 & 0,0 & 2,5 & 2,5 \\
\hline Total & $\mathbf{0 , 0}$ & $\mathbf{7 , 4}$ & $\mathbf{5 , 9}$ & $\mathbf{1 3 , 3}$ \\
\hline Promedio & $\mathbf{0 , 0}$ & $\mathbf{1 , 5}$ & $\mathbf{1 , 2}$ & $\mathbf{2 , 7}$ \\
\hline
\end{tabular}

Región de los Ríos Pequeño Propietario

\begin{tabular}{|c|r|r|r|r|}
\hline \multirow{2}{*}{ Año } & E. globulus & \multicolumn{1}{c|}{ E. nitens } & \multicolumn{1}{c|}{ Pino radiata } & \multicolumn{1}{c|}{ Total } \\
\cline { 2 - 5 } & \multicolumn{1}{c|}{ ( ha ) } & & & \\
\hline 2012 & 199,5 & 546,8 & 151,7 & 897,9 \\
\hline 2013 & 396,3 & 581,2 & 294,9 & $1.272,4$ \\
\hline 2014 & 235,1 & 477,8 & 274,6 & 987,5 \\
\hline 2015 & 26,9 & 138,6 & 157,5 & 323,0 \\
\hline 2016 & 313,9 & 693,1 & 131,4 & $1.138,3$ \\
\hline Total & $\mathbf{1 . 1 7 1 , 6}$ & $\mathbf{2 . 4 3 7 , 4}$ & $\mathbf{1 . 0 1 0 , 1}$ & $\mathbf{4 . 6 1 9 , 1}$ \\
\hline Promedio & $\mathbf{2 3 4 , 3}$ & $\mathbf{4 8 7 , 5}$ & $\mathbf{2 0 2 , 0}$ & $\mathbf{9 2 3 , 8}$ \\
\hline
\end{tabular}


Región de los Lagos Mediano Propietario

\begin{tabular}{|c|r|r|r|r|}
\hline \multirow{2}{*}{ Año } & E. globulus & \multicolumn{1}{c|}{ E. nitens } & \multicolumn{1}{c|}{ Pino radiata } & \multicolumn{1}{c|}{ Total } \\
\cline { 2 - 5 } & \multicolumn{5}{|c|}{ ( ha ) } \\
\hline 2012 & 115,8 & 209,7 & 0,0 & 325,5 \\
\hline 2013 & 0,0 & 0,0 & 0,0 & 0,0 \\
\hline 2014 & 0,0 & 0,0 & 0,0 & 0,0 \\
\hline 2015 & 56,5 & 0,0 & 0,0 & 56,5 \\
\hline 2016 & 0,0 & 0,0 & 47,8 & 47,8 \\
\hline Total & $\mathbf{1 7 2 , 3}$ & $\mathbf{2 0 9 , 7}$ & $\mathbf{4 7 , 8}$ & $\mathbf{4 2 9 , 8}$ \\
\hline Promedio & $\mathbf{3 4 , 5}$ & $\mathbf{4 1 , 9}$ & $\mathbf{9 , 6}$ & $\mathbf{8 6 , 0}$ \\
\hline
\end{tabular}

Región de los Lagos Pequeño Propietario

\begin{tabular}{|c|r|r|r|r|}
\hline \multirow{2}{*}{ Año } & E. globulus & \multicolumn{1}{|c|}{ E. nitens } & \multicolumn{1}{c|}{ Pino radiata } & \multicolumn{1}{c|}{ Total } \\
\cline { 2 - 5 } & \multicolumn{4}{|c|}{ ( ha ) } \\
\hline 2012 & 109,4 & 116,3 & 134,3 & 359,9 \\
\hline 2013 & 150,5 & 197,0 & 146,4 & 493,9 \\
\hline 2014 & 192,9 & 179,1 & 146,4 & 518,4 \\
\hline 2015 & 53,3 & 243,1 & 95,3 & 391,6 \\
\hline 2016 & 203,6 & 518,5 & 191,7 & 913,7 \\
\hline Total & $\mathbf{7 0 9 , 6}$ & $\mathbf{1 . 2 5 4 , 0}$ & $\mathbf{7 1 4 , 0}$ & $\mathbf{2 . 6 7 7 , 6}$ \\
\hline Promedio & $\mathbf{1 4 1 , 9}$ & $\mathbf{2 5 0 , 8}$ & $\mathbf{1 4 2 , 8}$ & $\mathbf{5 3 5 , 5}$ \\
\hline
\end{tabular}


APÉNDICE №2

RAZÓN DE REFORESTACIÓN POR REGIÓN, AÑO Y TIPO DE PROPIETARIO

Región de Valparaíso

\begin{tabular}{|c|c|c|}
\hline Año & Mediano Propietario & Pequeño Propietario \\
\hline 2012 & 13,5 & 23,4 \\
\hline 2013 & 61,1 & 19,6 \\
\hline 2014 & indefinido & 14,0 \\
\hline 2015 & indefinido & 0,0 \\
\hline 2016 & 1,5 & 4,4 \\
\hline
\end{tabular}

Región de O’Higgins

\begin{tabular}{|c|c|c|}
\hline Año & Mediano Propietario & Pequeño Propietario \\
\hline 2012 & 0,4 & 1,4 \\
\hline 2013 & 0,1 & 1,8 \\
\hline 2014 & 0,0 & 2,5 \\
\hline 2015 & 0,0 & 0,7 \\
\hline 2016 & 0,1 & 1,0 \\
\hline
\end{tabular}

Región del Maule

\begin{tabular}{|c|c|c|}
\hline Año & Mediano Propietario & Pequeño Propietario \\
\hline 2012 & 0,4 & 0,2 \\
\hline 2013 & 0,1 & 0,4 \\
\hline 2014 & 0,0 & 0,3 \\
\hline 2015 & 0,0 & 0,1 \\
\hline 2016 & 0,1 & 0,3 \\
\hline
\end{tabular}

Región del Bio Bio

\begin{tabular}{|c|c|c|}
\hline Año & Mediano Propietario & Pequeño Propietario \\
\hline 2012 & 0,5 & 1,3 \\
\hline 2013 & 0,2 & 1,6 \\
\hline 2014 & 0,1 & 1,5 \\
\hline 2015 & 0,3 & 0,7 \\
\hline 2016 & 1,3 & 1,5 \\
\hline
\end{tabular}


Región de la Araucanía

\begin{tabular}{|c|c|c|}
\hline Año & Mediano Propietario & Pequeño Propietario \\
\hline 2012 & 8,2 & 2,5 \\
\hline 2013 & 0,7 & 1,9 \\
\hline 2014 & 4,2 & 2,1 \\
\hline 2015 & 1,2 & 2,1 \\
\hline 2016 & 1,1 & 2,4 \\
\hline
\end{tabular}

Región de los Ríos

\begin{tabular}{|c|c|c|}
\hline Año & Mediano Propietario & Pequeño Propietario \\
\hline 2012 & indefinido & 4,9 \\
\hline 2013 & 2,2 & 3,3 \\
\hline 2014 & indefinido & 2,6 \\
\hline 2015 & indefinido & 1,1 \\
\hline 2016 & 0,0 & 7,7 \\
\hline
\end{tabular}

Región de los Lagos

\begin{tabular}{|c|c|c|}
\hline Año & Mediano Propietario & Pequeño Propietario \\
\hline 2012 & indefinido & 1,7 \\
\hline 2013 & indefinido & 2,4 \\
\hline 2014 & indefinido & 2,5 \\
\hline 2015 & indefinido & 3,1 \\
\hline 2016 & 0,0 & 3,8 \\
\hline
\end{tabular}

